\title{
Generation and analysis of the radioactive particles emitted from the Fukushima nuclear accident
}

\author{
M. INAGAKI ${ }^{1}$, R. SATO $^{2}$, S. SEKIMOTO ${ }^{1}$, \\ K. TAKAMIYA ${ }^{1}$, Y. OKI $^{1}$, AND T. OHTSUKI ${ }^{1}$
}

${ }^{1}$ Institute for Integrated Radiation and Nuclear Science, Kyoto University, 2-1010 Asashiro-nishi, Kumatori, Sennan, Osaka 590-0494, Japan (inagaki.makoto.2r@kyoto-u.ac.jp) ${ }^{2}$ Graduate School of Engineering, Kyoto University, Kyotodaigaku-katsura, Nishikyo-ku, Kyoto 615-8510, Japan

After the Fukushima Daiichi Nuclear Power Plant (F1NPP) accident, various kinds of radioactive materials were released to the surrounding environment. Among those materials, large amounts of glassy particles have been found and some particles include highly concentrated radiocaesium [1]. To study physical and chemical properties of those glassy particles can make us understand what happened in the nuclear reactors during the F1NPP accident. However, to our knowledge, there is not much information about that described above.

To improve our understanding about what happened in the nuclear reactors, glassy particles simulant materials were artificially produced and then, those simulants were subjected to chemical analysis.

The simulants were produced by heating a mixture of diatomaceous earth, which is one of the candidate for the source material of glassy particles, and artificial seawater. Then, those simulants produced were studied by SEM-EDS for surface analysis and by neutron activatioin analysis (NAA) using Kyoto university reactor for investigating bulk elemental composition. How to produce the simulants and the results obtained by SEM-EDS and NAA will be reported in the presentation

[1] Igarashi et al. (2019) J. Environ. Radioact. 205-206, 101118. 